\title{
Superfluid density in the two-dimensional attractive Hubbard model: Quantitative estimates
}

\author{
P. J. H. Denteneer \\ Instituut-Lorentz, University of Leiden, P.O. Box 9506, 2300 RA Leiden, The Netherlands
}

(Received 23 June 1993)

\begin{abstract}
A nonzero superfluid density is equivalent to the occurrence of a Meissner effect and therefore signals superconductivity. A recent theorem shows that in the case of a spectrum with a gap the superfluid density is equivalent to the Drude weight. This theorem is employed to compare approximate calculations of the superfluid density in the two-dimensional attractive Hubbard model using the Hartree-Fock approximation with exact diagonalization calculations of the Drude weight. Direct comparison of the approximate results with recent finite-temperature quantum Monte Carlo calculations is also made. The approximate results are found to be quantitatively accurate for all fillings, except close to half-filling.
\end{abstract}

The superfluid density is the characteristic quantity in describing superfluid or superconducting order of physical systems. Experimentally, it is observed as the portion of the total density which is not susceptible to mechanical drag. Theoretically, it can be introduced in a variety of ways: the proportionality constant in the incremental free energy upon twisting the order parameter, ${ }^{1}$ the linear response to a twisted boundary condition, ${ }^{2}$ or it can be related to a limit of the current-current correlation function. ${ }^{3,4}$ In a system in a superconducting state the superfluid density is inversely proportional to the square of the penetration depth of magnetic lines of force. A nonzero superfluid density therefore corresponds to a Meissner effect. ${ }^{4}$ In two dimensions, the transition to the ordered state is of the Kosterlitz-Thouless type and the superfluid density exhibits a universal jump at the transition. ${ }^{5}$ Knowledge of the superfluid density is therefore important to decide whether a system is in a superfluid (superconducting) state and can also be used to estimate the critical temperature. ${ }^{6}$

The Hubbard model is a simplified model for interacting electrons on a lattice. The electrons hop between lattice sites and experience each others presence, besides the effect of the Pauli exclusion principle, only if two electrons of opposite spin occupy the same site. Since the model includes both charge and spin degrees of freedom, it may be useful to understand the behavior of materials in which both magnetism and superconductivity can occur. Examples of such materials are high-temperature superconductors and heavy fermion materials. Although a connection with these materials is more likely to be found in the Hubbard model with on-site repulsion (because it is an antiferromagnetic insulator for a density of one electron per site like the undoped copper oxides), it is of interest (see below) to study the "negative-U" Hubbard model, which has on-site attraction. The Hamiltonian for the attractive Hubbard model is given by

$$
\mathcal{H}=-\sum_{i j \sigma} t_{i j} c_{i \sigma}^{\dagger} c_{j \sigma}+U \sum_{i} n_{i \uparrow} n_{i \downarrow}-\mu \sum_{i \sigma} n_{i \sigma}
$$

where $c_{i \sigma}^{\dagger}$ creates an electron at site $i$ with $\operatorname{spin} \sigma, n_{i \sigma}=$ $c_{i \sigma}^{\dagger} c_{i \sigma}, t_{i j}$ is the one-electron transfer integral between sites $j$ and $i$ ( $t_{i j}$ equals $t$ if $i$ and $j$ are nearest neighbors and 0 otherwise), $U$ the on-site attraction $(U<0)$, and $\mu$ the chemical potential ( $\mu=U / 2$ corresponds to a halffilled lattice, i.e., $\sum_{i \sigma}\left\langle n_{i \sigma}\right\rangle=1$ ).

The attractive Hubbard model on a (two-dimensional) square lattice is found to have superconducting order, ${ }^{7}$ probably for the whole range of interaction constants. This model therefore opens the possibility of comparing approximate calculations (for instance using trial wave functions) of the superfluid density (to be denoted by $\rho_{s}$ in the following) with more exact results, for instance obtained using exact diagonalization of the Hamiltonian on small clusters as well as quantum Monte Carlo (QMC) calculations. Such a comparison is the purpose of this paper and has become possible through an important recent paper by Scalapino et al. ${ }^{4}$ Besides containing results of QMC calculations of $\rho_{s}$ at very low temperatures, it proves the theorem that the superfluid weight $D_{s}$ (related to $\rho_{s}$ by $\left.\rho_{s}=D_{s} / 4 \pi e^{2}\right)$ and the Drude weight $D$ are equal if there is a gap in the spectrum. The theorem therefore enables comparison of approximate results for $\rho_{s}$ with recent results of exact diagonalization studies of $D$ on small lattices. ${ }^{8,9}$ Scalapino et al. obtain expressions for $D_{s}$ and $D$ in terms of different limits of the currentcurrent correlation function:

$$
\begin{aligned}
& \frac{D_{s}}{\pi e^{2}}=\left\langle-k_{x}\right\rangle-\Lambda_{x x}\left(q_{x}=0, q_{y} \rightarrow 0, i \omega_{m}=0\right) \\
& \frac{D}{\pi e^{2}}=\left\langle-k_{x}\right\rangle-\Lambda_{x x}\left(\mathbf{q}=0, i \omega_{m} \rightarrow 0\right)
\end{aligned}
$$

For two dimensions, $\left\langle k_{x}\right\rangle$ is half the kinetic energy per site and $\Lambda_{x x}(\mathbf{q}, \omega)$ is the double Fourier transform of the current-current correlation function. The theorem is thus equivalent to the statement that if there is a gap in the spectrum the order in which $q_{y}$ and $i \omega_{m}$ go to zero may be interchanged. For further details, we refer to Ref. 4. In a previous paper, we presented calculations of $\rho_{s}$ in the Hartree-Fock approximation (HFA), as well as variational Monte Carlo calculations using a Gutzwiller projected trial wave function. ${ }^{10}$ The superfluid density $\rho_{s}$ is 
calculated as a second derivative of the free energy per site $f$ with respect to a phase twist $\phi$ of the (complex) order parameter $\Delta: f(\phi)=f(0)+\frac{1}{2} \rho_{s} \phi^{2}+\mathcal{O}\left(\phi^{4}\right)$ for $\Delta(\mathbf{r})=|\Delta| e^{i \mathbf{q} \cdot \mathbf{r}}$ with $\mathbf{q}=(\phi, 0)$. We found that the variational Gutzwiller Monte Carlo calculation increased the values of $\rho_{s}$ only by typically $5 \%$. Because of this small difference and because, using the HFA, $\rho_{s}$ can be found easily as a function of $U / t$, density $n$, and temperature $T$, in the following we will only consider the HFA results. The expression for $\rho_{s}$ as a function of $U / t, n$, and $T$ is a sum over the Brillouin zone of the square lattice. The formula was given before $\mathrm{e}^{6,10}$ and is not repeated here; we just note that it can be evaluated both on finite lattices and in the thermodynamic limit. In the HFA at $T=0$, $\rho_{s}$ is given by just the kinetic energy term in (2) (see Refs. 4 and 10); comparison with exact results will thus reveal the importance of current-current correlations.

First, we compare our Hartree-Fock calculations of $\rho_{s}$ with the results for the Drude weight $D$ from exactly diagonalizing the Hubbard Hamiltonian using Lanczos techniques. $^{8}$ The latter results were obtained on $4 \times 4$ lattices and are for temperature $T=0$. The HFA calulations of $\rho_{s}$ are extensively described in Ref. 10. In Fig. $1, D / 2 \pi e^{2}$ is compared to two times $\rho_{s}$ for $U / t=-4,-8,-10$, and -20 . We have to multiply our results for $\rho_{s}$ as obtained in Ref. 10 by a factor of 2 in order to compare with $D / 2 \pi e^{2}$. This calibration is most easily obtained by considering the $U / t \rightarrow 0$ limit on an infinitely large lattice. In this limit, at half-filling, $D / 2 \pi e^{2}$ is exactly $4 / \pi^{2},{ }^{8}$ whereas $\rho_{s}$ is exactly $2 / \pi^{2} .{ }^{10}$ For a better comparison, the HFA results are also computed on a

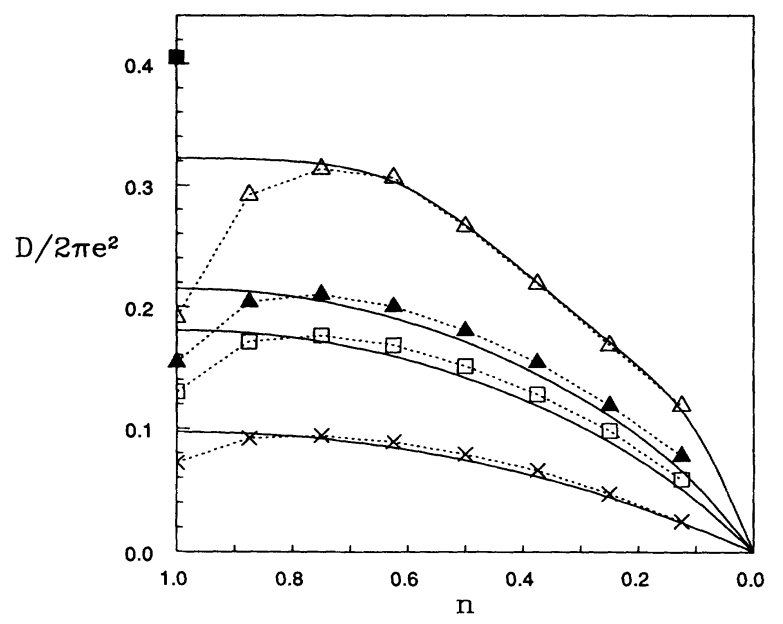

FIG. 1. Drude weight $D / 2 \pi e^{2}$ as a function of density $n$ for the negative- $U$ Hubbard model on a square lattice obtained by exact diagonalization on $4 \times 4$ lattices compared to two times the superfluid density $\rho_{s}$ calculated using the Hartree-Fock approximation (for comparison also computed on a $4 \times 4$ lattice using periodic boundary conditions in both directions). The open triangles, full triangles, open squares, and crosses denote the exact diagonalization results for $U / t=-4,-8,-10$, and -20 , respectively. The drawn lines are the corresponding Hartree-Fock results. The full square denotes the exact $U / t=0$ result in the thermodynamic limit, $4 / \pi^{2}$. Dotted lines are guides to the eye.
$4 \times 4$ lattice, using periodic boundary conditions in both the $x$ and $y$ directions. Only for $U / t=-4$ (or closer to 0 ) going to larger lattices or changing the boundary conditions (for instance to periodic in the $x$ direction and antiperiodic in the $y$ direction) would visibly affect the curves in Fig. 1; however, not in a very significant way. Although not explicitly stated there, we assume that the exact diagonalization results were obtained using periodic boundary conditions. ${ }^{11}$

Two important conclusions may be drawn from Fig. 1. In the first place, the approximate Hartree-Fock results agree surprisingly well with the exact results for densities $n<0.8$. Only for densities close to half-filling the agreement is not very good and the approximate result misses the qualitative feature of an initial rise of $\rho_{s}$ when going off half-filling. We observe that for decreasing (absolute) values of $U / t$ the differences close to half-filling get larger. We conclude that only close to half-filling the contribution of the current-current correlation function, which is neglected when making the HFA, becomes significant. Second, even at half-filling the exact result for $D$ does not vanish, implying a nonzero superfluid density at $T=0$. However, since the Hubbard model at halffilling has the full Heisenberg symmetry, it must have a critical temperature $T_{c}$ for the onset of long-ranged order equal to zero (Mermin-Wagner theorem). Because the HFA breaks down the Heisenberg symmetry to $X Y$ symmetry, it incorrectly renders a finite $T_{c}$, when invoking the Kosterlitz-Thouless universal jump relation for $\rho_{s}{ }^{6,10}$ However, as becomes clear from the comparison in Fig. 1, the value of $\rho_{s}$ at $T=0$ obtained in the HFA never differs from the exact result by more than a factor of 2 (for the values of $U / t$ considered). Therefore the estimate of $\rho_{s}$ in the HFA is not so much incorrect as is its temperature dependence: any nonzero $T$ will make the exact $\rho_{s}$ vanish, but a finite value at $T=0$ is allowed.

We further note that, because negative- $U$ and positive$U$ Hubbard models at half-filling can be mapped onto each other, thereby interchanging spin and charge degrees of freedom, the exact diagonalization results have implications for the positive- $U$ Hubbard model at halffilling as well. In particular, the spin stiffness associated with the antiferromagnetic order that the positive- $U$ Hubbard model has at half-filling ${ }^{12}$ maps onto $\rho_{s}$ (Ref. 13) and is given by $0.096,0.077,0.065$, and 0.036 for $U / t=4,8,10$, and 20, respectively (see Fig. 1). Corresponding HFA values are $0.161,0.108,0.090$, and 0.049 . In the limit $U / t \rightarrow \infty$ the HFA gives $0.25 J$ for $\rho_{s}$ (with $\left.J=4 t^{2} / U\right)$, which is the linear spin-wave approximation result for the $S=1 / 2$ Heisenberg antiferromagnet. ${ }^{14,15}$ We note that for $U / t=20$, the exact diagonalizations give $\rho_{s}=0.18 J$, which is exactly the result from series expansions for the $S=1 / 2$ Heisenberg antiferromagnet, $\rho_{s} / J=0.18 \pm 0.01 .^{14,16}$

The second comparison that can be made is that of our Hartree-Fock calculations of $\rho_{s}$ with the quantum Monte Carlo calculations of the superfluid weight $D_{s}$ for the negative- $U$ Hubbard model. ${ }^{4}$ The latter calculations were done with a finite, but very low, temperature $(T=0.1 t)$ on an $8 \times 8$ lattice for $U / t=-4$. In the QMC calculations, following (2), half the average kinetic energy per 
site, $\left\langle k_{x}\right\rangle$, is computed as well as the current-current correlation function $\Lambda_{x x}(\mathbf{q}, \omega)$. Because of the finite lattice on which the computations are performed the correlation function is only obtained for a discrete set of values of $\mathbf{q}$, the smallest of which has length $q=2 \pi / L$, where $L$ is the linear size of the lattice ( $L=8$ in Ref. 4$)$. The required limit $q_{y} \rightarrow 0$ in (2) can only be found by going to larger lattices or from an (uncontrolled) extrapolation using the values at $q_{y}=\pi / 2$ and $q_{y}=\pi / 4$. In Fig. 2 , the HFA result for two times $\rho_{s}$ is compared to the QMC results. For a good comparison, the HF result is calculated on an $8 \times 8$ lattice and for $T=0.1 t$ just as the QMC result; the change to the curve would however be barely visible if computed for a much larger lattice and $T=0$. The QMC results are extracted from Figs. 8 and 10 of Ref. 4 for five densities. We plot three quantities: the kinetic energy contribution to $D_{s} / 2 \pi e^{2}$ (octagons), the full $D_{s} / 2 \pi e^{2}$ as obtained from the smallest $q_{y}(=\pi / 4)$ possible on the lattice (crosses), and the full $D_{s} / 2 \pi e^{2}$ as obtained from extrapolating $\Lambda_{x x}(\mathbf{q}, \omega=0)$ to $q_{y}=0$ (triangles). The crosses correspond to the quantity plotted in Fig. 11 of Ref. 4, apart from a factor of $2 .{ }^{17}$ From Fig. 2 we see that the HFA result for two times $\rho_{s}$, which only contains the kinetic energy contribution, agrees very well with the kinetic energy from QMC. This means that in the HFA the kinetic energy is very well described quantitatively. We furthermore see that the extrapolated result for $\Lambda_{x x}(\mathbf{q}, \omega=0)$ brings $D_{s} / 2 \pi e^{2}$ in better agreement with the HFA result than the values obtained for $q_{y}=\pi / 4$. In this respect, it is also worth mentioning that in the QMC calculations $\Lambda_{x x}\left(q_{x}=0, q_{y}=\pi / 4, i \omega_{m}=0\right)$ is still somewhat temperature dependent (see Fig. 8 of Ref. 4) and will decrease when lowering the temperature further, thereby improving the agreement with the HFA result.

Finally, we note that, comparing Figs. 1 and 2, there is qualitative agreement between QMC results and exact diagonalization studies. Quantitative agreement is best if we use the extrapolated current-current correlation function from the QMC calculations. Quantitative agreement is likely to improve if the QMC calculations are performed for lower temperatures. If the finite-size effects in the exact diagonalization studies are similar to those in the HFA, going to larger lattices (which is hardly possible with present-day computers) will not seriously affect the exact diagonalization results for $\rho_{s}$.

In summary, we have shown that a key quantity in studying systems that may exhibit superconducting or

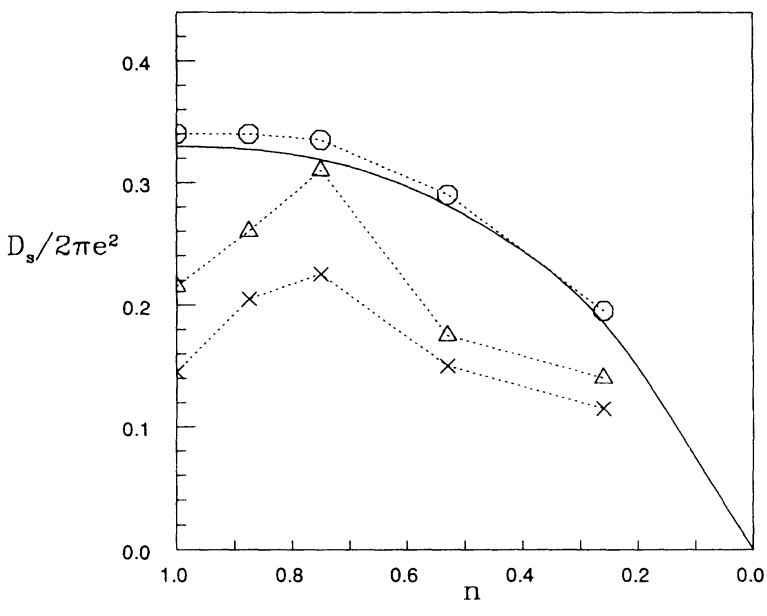

FIG. 2. Superfluid weight $D_{s} / 2 \pi e^{2}$ as a function of density $n$ for the negative- $U$ Hubbard model on a square lattice obtained from quantum Monte Carlo (QMC) calculations on an $8 \times 8$ lattice for temperature $T=0.1 t$ and $U / t=-4$ compared to two times the superfluid density $\rho_{s}$ calculated using the Hartree-Fock approximation (for comparison also computed for $T=0.1 t$ on an $8 \times 8$ lattice using periodic boundary conditions in both directions). The octagons denote the QMC result for the kinetic energy term contributing to $D_{s}$, whereas the crosses and triangles denote the QMC results for $D_{s} / 2 \pi e^{2}$ if the current-current correlation function is evaluated for the smallest $q_{y}$ attainable on an $8 \times 8$ lattice $\left(q_{y}=\pi / 4\right)$ or extrapolated to $q_{y}=0$, respectively (see text). The drawn line is the Hartree-Fock result. Dotted lines are guides to the eye.

superfluid order, the superfluid density, for the twodimensional attractive Hubbard model, is described surprisingly well in the Hartree-Fock approximation by comparing with exact diagonalization and quantum Monte Carlo calculations. Only for densities close to half-filling the quantitative agreement is not very good, implying that for such densities the contribution from the currentcurrent correlation function significantly reduces $\rho_{s}$, although not to the extent that $\rho_{s}$ vanishes at half-filling.

I acknowledge valuable contributions to the research presented here by J. M. J. van Leeuwen and discussions with K. S. Bedell.
${ }^{1}$ M.E. Fisher, M.N. Barber, and D. Jasnow, Phys. Rev. A 8, 1111 (1973).

${ }^{2}$ B.S. Shastry and B. Sutherland, Phys. Rev. Lett. 65, 243 (1990).

${ }^{3}$ D. Forster, Hydrodynamic Fluctuations, Broken Symmetry, and Correlation Functions (W.A. Benjamin, Reading, 1975).

${ }^{4}$ D.J. Scalapino, S.R. White, and S.C. Zhang, Phys. Rev. Lett. 68, 2830 (1992); Phys. Rev. B 47, 7995 (1993).
${ }^{5}$ D.R. Nelson, in Fundamental Problems in Statistical Mechanics $V$, edited by E.G.D. Cohen (North-Holland, Amsterdam, 1980).

${ }^{6}$ P.J.H. Denteneer, Guozhong An, and J.M.J. van Leeuwen, Europhys. Lett. 16, 5 (1991); 16, 509(E) (1991).

${ }^{7}$ R.T. Scalettar, E.Y. Loh, J.E. Gubernatis, A. Moreo, S.R. White, D.J. Scalapino, R.L. Sugar, and E. Dagotto, Phys. Rev. Lett. 62, 1407 (1989); A. Moreo and D.J. Scalapino, ibid. 66, 946 (1991). 
${ }^{8}$ E. Dagotto, A. Moreo, F. Ortolani, J. Riera, and D.J. Scalapino, Phys. Rev. B 45, 10107 (1992).

${ }^{9}$ D.J. Scalapino, Physica C 185-189, 104 (1991).

${ }^{10}$ P.J.H. Denteneer, Guozhong An, and J.M.J. van Leeuwen, Phys. Rev. B 47, 6256 (1993).

${ }^{11}$ We note that exact diagonalization studies of $D$ from the same group reported earlier (Ref. 9) obtained significantly different results from those of Ref. 8 for $U / t=-4$ for densities close to half-filling. The discrepancy could be due to different boundary conditions.

12 J.E. Hirsch and S. Tang, Phys. Rev. Lett. 62, 591 (1989).

${ }^{13}$ For the positive- $U$ Hubbard model at half-filling both $D$ and $D_{s}$ vanish, the model being in an (antiferromagnetic) insulating phase.

${ }^{14}$ E. Manousakis, Rev. Mod. Phys. 63, 1 (1991).

${ }^{15}$ P.J.H. Denteneer and J.M.J. van Leeuwen, Europhys. Lett. 22, 413 (1993).

${ }^{16}$ R.R.P. Singh, Phys. Rev. B 39, 9760 (1989).

${ }^{17}$ In Fig. 11 of Ref. 4 a plotting error concerning the density axis appears to have been made: to be consistent with Figs. 8 and 10 the highest-density data point is for half-filling. The other data points should correspond to the densities of Figs. 8 and 10. 